\title{
Contour Tracking When Two Gray-Level Discontinuities Are Close to Each Other
}

\author{
Marcello Demi ${ }^{1,2}$, Elisabetta Bianchini ${ }^{1}$, Francesco Faita ${ }^{1}$, \\ and Vincenzo Gemignani ${ }^{1}$ \\ ${ }^{1}$ CNR Institute of Clinical Physiology, via G.Moruzzi 1, 56124 Pisa, Italy \\ ${ }^{2}$ Esaote $\mathrm{SpA}$, via di Caciolle 15, 50127 Firenze, Italy \\ \{marcello.demi, elisabetta.bianchini, francesco.faita, \\ vincenzo.gemignani\}@ifc.cnr.it
}

\begin{abstract}
Vascular measurements are indispensable to quantify important indexes of cardiovascular risk and image processing systems are needed to automatically track the vascular structures through sequences of echographic images. Given a starting contour $c_{n}$ on frame $f_{n}$, a contour tracking algorithm is generally based on the application of a mathematical operator at the points of $c_{n}$ and on an iterative procedure which brings such points to the respective points of the contour $c_{n+1}$ on the subsequent frame $f_{n+1}$. In this paper, the performances of a mathematical operator which looks for similar regional gray level distributions are compared to those of an edge detection operator. The paper shows that when two or more gray-level discontinuities are present and close to each other, as in the case of arteries, both operators should be used sequentially.
\end{abstract}

Keywords: Contour Tracking, Vascular Images, Ultrasound Imaging.

\section{Introduction}

From a conceptual point of view, the problem of locating and tracking a gray level discontinuity through a sequence of images is very simple: given a contour on a frame, the task is reduced to locating the respective contour on the subsequent frame. Once a contour is determined on the $\mathrm{n}^{\text {th }}$ frame, the simplest strategy is to use it as a starting contour to locate the nearest contour on the $(n+1)^{\text {th }}$ frame. Given an approximate starting contour, methods have been developed which looks for the nearest contour on vascular images [1]. This strategy, however, can fail when two or more discontinuities are present and close to each other [2]. In this case the position of the starting contour is not sufficient to discriminate the target contour on the $(n+1)^{\text {th }}$ frame. Information on the regional gray level distribution of the $\mathrm{n}^{\text {th }}$ frame at the points of the starting contour is needed to discriminate points of the $(n+1)^{\text {th }}$ frame with similar regional gray level distributions. Therefore, technically, two different localization strategies are delineated. However, the second strategy usually is not used separately even though this is possible: commonly, an optimization procedure is used to locate contours which represent a good compromise between a measure of similarity and a measure of edgeness [2][3]. In this paper two mathematical operators, which well represent the two localization strategies mentioned above, are analysed 
and the results of our analysis, which were obtained on test sequences of synthetic images and on echographic images of carotid arteries, are illustrated and discussed. The paper shows why, when either long sequences of images are considered or when two gray level discontinuities are present and close to each other, as in the case of arteries, both operators should be used sequentially.

\section{Contour Tracking by Using Vector $b$}

Given a function $f(x, y, t)$, describing a gray level image map at time $t$, the first absolute central moment $e(x, y, t)$ and vector $\mathbf{b}(x, y, t)$ can be defined

$$
\begin{gathered}
e(x, y, t)=\int_{-\infty}^{\infty} \int_{-\infty}^{\infty} h\left(x, y, t, \tau_{x}, \tau_{y}\right) d \tau_{x} d \tau_{y} \\
\mathbf{b}(x, y, t)=\left\{\begin{array}{cc}
\frac{1}{e(x, y, t)} \int_{-\infty}^{\infty} \int_{-\infty}^{\infty} h\left(x, y, t, \tau_{x}, \tau_{y}\right) \Gamma d \tau_{x} d \tau_{y} & e(x, y, t) \neq 0 \\
0 & e(x, y, t)=0
\end{array}\right. \\
h\left(x, y, t, \tau_{x}, \tau_{y}\right)=\left|f_{1}(x, y, t)-f\left(x-\tau_{x}, y-\tau_{y}, t\right)\right| g\left(\tau_{x}, \tau_{y}, \sigma_{2}\right) \\
f_{1}(x, y, t)=\int_{-\infty}^{\infty} \int_{-\infty}^{\infty} f\left(x-\tau_{x}, y-\tau_{y}, t\right) g\left(\tau_{x}, \tau_{y}, \sigma_{1}\right) d \tau_{x} d \tau_{y}
\end{gathered}
$$

where $g\left(x, y, \sigma_{l}\right)$ and $g\left(x, y, \sigma_{2}\right)$ are two Gaussian functions with apertures $\sigma_{l}$ and $\sigma_{2}$, and $\Gamma$ is a vector of components $-\tau_{x},-\tau_{y}$. Two properties of vector $\mathbf{b}$ must be outlined when a starting point $p$ close to a gray level discontinuity is given: i) vector $\mathbf{b}$ is normal to the discontinuity and ii) if a configuration with $\sigma_{l}>\sigma_{2} / \pi$ is chosen then $\mathbf{b}$ joins a point $p^{\prime}$ which is closer to the discontinuity than the starting point $p$ [4]. Consequently, if a configuration with $\sigma_{1}>\sigma_{2} / \pi$ is chosen, vector $\mathbf{b}$ can be applied recursively at every point of a starting contour, which is given on the image map $f(x, y, t)$, to find the edge points of the contour $c$ we are looking for at time $t$. Once contour $c$ is located, then every point $p_{i}$ of $c$ can be used as a starting point on the image map $f(x, y, t+\Delta t)$ to find the respective point $p_{i}{ }^{\prime}$ of contour $c^{\prime}$ at time $t+\Delta t$ thus estimating, in this way, the displacements of points $p_{i}$. Obviously, according to the previous property i), only the component of the displacement which is normal to the edge is estimated.

It is worth noting, however, that the above algorithm does not work when two different discontinuities, with two different contours $c_{A}$ and $c_{B}$, exist on $f(x, y, t)$ and the distance between them is comparable to the magnitude of the displacement. In this case, once contour $c_{A}$ is located on frame $t$, points of $c_{A}$ can be attracted from the discontinuity $c_{B}^{\prime}$ of frame $t+\Delta t$ when using $c_{A}$ as the starting contour to locate $c_{A}^{\prime}$. That is, given a 
contour $c$ on frame $t$, vector $\mathbf{b}$ simply locates the closest contour $c^{\prime}$ on frame $t+\Delta t$ and, under particular conditions, $c^{\prime}$ may be not the contour we are looking for.

\section{Contour Tracking by Using the Optical-Flow Vector}

The optical-flow vector shows properties that make it potentially able to overcome the problem illustrated in the previous section. Given the function $f(x, y, t)$ the optical-flow equation is based on the assumption that the distribution of the gray levels does not change significantly during a short time interval

$$
\begin{gathered}
f(x, y, t) \approx f(x+\Delta x, y+\Delta y, t+\Delta t) \\
f_{x} \hat{u}+f_{y} \hat{v}+f_{t}=0 \\
\hat{\mathbf{w}}=-\frac{f_{t}}{|\nabla f|^{2}} \nabla f .
\end{gathered}
$$

If the Taylor series expansion of the second member of eq.(5) is computed at point $(x, y, t)$, then the canonical optical-flow eq.(6) is obtained when the series expansion is limited to the first order [5]. Here the unknown $\hat{u}$ and $\hat{v}$ are the estimated components of the velocity $\mathbf{w}$ which is experienced by the image map at point $p \equiv(x, y)$ at instant $t$, and the velocity $\mathbf{w}$ can be estimated by computing eq.(7).

Usually every frame of the image sequence is convolved with a low-pass filter to cope with noise and both the temporal derivative $f_{t}$ and the spatial gradient $\nabla f$ are computed as finite differences. Eq.(7) can be correctly used to track contours through image sequences since in this case it is applied at edge points and, consequently, the magnitude of $\nabla f$ is different from zero. Once a contour $c$ is located on the image map $f(x, y, t)$, eq.(7) can be computed at every point $p_{i}$ of $c$ in order to find the points $p_{i}{ }^{\prime}$ with a similar gray level on the image map $f(x, y, t+\Delta t)$ thus estimating the displacements $\Delta \mathbf{s}=\mathbf{w} \Delta t$ of points $p_{i}$ and locating the subsequent contour $c^{\prime}$ at time $t+\Delta t$. It is worth noting that, according to the hypothesis introduced with eq.(6), eq.(7) provides a proper estimate of $\Delta \mathbf{s}$ only in the case of small displacements. However, when larger displacements are considered eq.(7) can still be used: it can be applied iteratively on the same frame until a convergence condition is satisfied. Obviously, due to the aperture problem only the component of the displacement which is perpendicular to the spatial discontinuity can be estimated.

As we have seen above, the strategy based on vector $\mathbf{b}$ shows its weakness when tracking contours of complex structures and the optical-flow operator may be helpful to overcome such a problem. It is worth noting, however, that also the latter strategy shows intrinsic limitations that do not render it self-sufficient. According to eq.(5), the optical-flow vector looks for a gray level distribution which is similar to the gray level distribution associated with the starting point located on the previous frame. The central remark is that this mathematical operator does not look for edge points. Even if the starting point is an edge point, the optical-flow operator, by definition, looks for something which is similar and this may not necessarily be an edge point. Moreover, 
in real work conditions inevitable small localization errors propagate through the sequence and they are unrecoverable.

\section{Test Planning and Results}

The figure of merit defined by Pratt [6] was used as a measure of performance:

$$
R=\frac{100}{\operatorname{MAX}\left(I_{A}, I_{I}\right)} \sum_{i=1}^{I_{A}} \frac{1}{1+a d_{i}^{2}}
$$

In eq.(8) $I_{A}$ and $I_{I}$ represent the number of the actual and ideal edge points, respectively; $a$ is a scaling constant; and $d_{i}$ is the distance between the $\mathrm{i}^{\text {th }}$ actual edge point, which is located by the contour tracking procedure, and the nearest ideal edge point. A constant $a$ equal to 0.25 is used, thus giving rise to $R$ values of $80,50,31,20$ when localization errors $d_{i}$ of $1,2,3,4$ pixels, respectively, occur. Let $\sigma_{1}, \sigma_{2}$ and $\sigma$ be the apertures of the first absolute central moment and the aperture of the Gaussian filter which is used by the optical-flow operator. A starting contour of 1000 points is given on the first frame of test sequences of 10,20,40,60,80 and 100 frames and the Pratt index $R$ is computed on the final contour which is located on the last frame of the sequence. The actual edge points were located with iterative procedures both when using the $\mathbf{b}$ and the optical-flow operators. The iterative procedures stop when the point obtained with the $j^{\text {th }}$ iteration is the same point determined either by the $(j-1)^{t h}$ iteration or by the $(j-2)^{t h}$ iteration. The last point is marked as an actual edge point only if its distance from the ideal discontinuity is less than $\sigma_{2}$ and $\sigma$ pixels, respectively. The procedures also stop when do not converge in 10 iterations and, in this case, the last point is not marked as an actual edge point. The contour which is located on the frame $f_{n}$ is used as starting contour to locate the contour on frame $f_{n+1}$. Two tests were performed to highlight two different problems.

\subsection{Isolated Gray-Level Discontinuity}

The first test sequence includes a moving step discontinuity affected by additive white Gaussian noise. The aim of this test is to place emphasis on the localization failures of a contour tracking procedure based on the optical-flow operator. Table 1 shows how $R$ varies when using the two different strategies, when varying the displacement $\Delta s$ of the gray level discontinuity between two consecutive frames and when varying the number of frames of the sequence from 10 to 100 . The results of table 1 were obtained with a Gaussian noise of variances $v^{2}$ of $400 \mathrm{i.u.}{ }^{2}$; a contrast at the step discontinuity of 50 i.u.; and apertures $\sigma_{1}=2 \sigma_{2}=6$ and $\sigma=6$ pixels. Analogous results were, however, obtained when these parameters were varied. Obviously, better results are obtained when increasing the $S N R$ and the apertures $\sigma_{1} \sigma_{2}$ and $\sigma$, but the localization problems of the two operators do not disappear unless ideal Gaussian function $g\left(x, y, \sigma_{i}\right)$ of infinite width and noise free test discontinuities are considered: they are only postponed to longer sequences and to greater displacements. 
Table 1. The table shows how $R$ decreases when both the optical-flow operator $(\mathbf{O F})$ is used and when the number of frames of the sequence increases. The Table also shows how $R$ decreases to zero when $\Delta s>2 \sigma_{2}$ pixels if the $\mathbf{b}$ operator is used.

\begin{tabular}{|c|c|c|c|c|c|c|c|c|c|c|c|c|}
\hline$\Delta \mathbf{s}$ & $\mathbf{O F}_{10}$ & $\mathbf{b}_{10}$ & $\mathbf{O F}_{20}$ & $\mathbf{b}_{20}$ & $\mathbf{O F}_{40}$ & $\mathbf{b}_{40}$ & $\mathbf{O F}_{60}$ & $\mathbf{b}_{60}$ & $\mathbf{O F}_{80}$ & $\mathbf{b}_{80}$ & $\mathbf{O F}_{100}$ & $\mathbf{b}_{100}$ \\
\hline 2 & 86 & 99 & 77 & 98 & 64 & 97 & 52 & 95 & 43 & 93 & 34 & 92 \\
\hline 4 & 86 & 100 & 77 & 99 & 63 & 98 & 51 & 96 & 43 & 95 & 34 & 94 \\
\hline 6 & 87 & 100 & 76 & 99 & 62 & 98 & 51 & 97 & 42 & 95 & 32 & 95 \\
\hline 8 & 85 & 100 & 77 & 99 & 62 & 97 & 52 & 95 & 41 & 94 & 32 & 92 \\
\hline 10 & 85 & 98 & 75 & 94 & 60 & 91 & 47 & 88 & 38 & 84 & 30 & 80 \\
\hline 12 & 84 & 10 & 67 & 1 & 55 & 0 & 42 & 0 & 34 & 0 & 27 & 0 \\
\hline
\end{tabular}

The table shows how the localization failures increase when the optical-flow operator $(\mathbf{O F})$ is used and how the errors increase when the number of frames of the sequence increases from 10 to 100 . The point is that two matching edges may have different gray levels. This is not a problem when a localization strategy based on the $\mathbf{b}$ operator is used since the procedure looks for the nearest edge point and the result does not depend on its gray level. By contrast, the localization strategy based on the optical-flow is penalized since the procedure looks for a point with a similar gray level and this may be not an edge point. Moreover, the localization errors propagate through the sequence and, consequently, the magnitude of the final error increases when the number of frames of the sequence increases. Table 1 also shows that, when the magnitude of the displacement $\Delta s$ is greater than 10 pixels, the errors increase dramatically if the $\mathbf{b}$ operator is used. This is caused by the fact that in this case the aperture $\sigma_{2}$ is small with respect to $\Delta s$ and the operator $\mathbf{b}$ cannot be attracted from the gray level discontinuity of frame $f_{n+1}$ when it is applied to points of the contour of frame $f_{n}$. By contrast, the optical-flow strategy still works when vector $\mathbf{b}$ fails and continues to work when $\Delta s$ increases since the sign of the temporal derivative in eq.(7) does not change and, consequently, the iterative procedure still joins a starting point $p$ of frame $f_{n}$ to the respective point $p$ ' of frame $f_{n+1}$.

\subsection{Complex Gray-Level Discontinuity}

The second test sequence includes a moving discontinuity shaped as a rect function affected by additive white Gaussian noise. The aim of this test is to highlight the localization failures derived from a low frame rate. Table 2 shows how $R$ varies when increasing the displacement $\Delta s$ of the gray level discontinuity between two consecutive frames, that is, when decreasing the frame rate of the sequence.

Table 2. The table shows that the optical-flow strategy still works when vector $\mathbf{b}$ fails

\begin{tabular}{|c|c|c|c|c|c|c|c|c|c|c|c|c|}
\hline$\Delta \mathbf{s}$ & $\mathbf{O F}_{10}$ & $\mathbf{b}_{10}$ & $\mathbf{O F}_{20}$ & $\mathbf{b}_{20}$ & $\mathbf{O F}_{40}$ & $\mathbf{b}_{40}$ & $\mathbf{O F}_{60}$ & $\mathbf{b}_{60}$ & $\mathbf{O F}_{80}$ & $\mathbf{b}_{80}$ & $\mathbf{O F}_{100}$ & $\mathbf{b}_{100}$ \\
\hline 2 & 93 & 100 & 85 & 100 & 63 & 100 & 48 & 100 & 35 & 100 & 27 & 100 \\
\hline 4 & 97 & 50 & 88 & 21 & 60 & 5 & 45 & 2 & 34 & 0 & 24 & 0 \\
\hline 6 & 95 & 0 & 83 & 0 & 57 & 0 & 41 & 0 & 30 & 0 & 26 & 0 \\
\hline 8 & 75 & 0 & 48 & 0 & 29 & 0 & 19 & 0 & 13 & 0 & 8 & 0 \\
\hline 10 & 0 & 0 & 0 & 0 & 0 & 0 & 0 & 0 & 0 & 0 & 0 & 0 \\
\hline
\end{tabular}


The table shows how the localization failures increase when $\Delta s$ increases both when using the optical-flow and the $\mathbf{b}$ operator. Given a rect function, which is $A$ pixels wide in absence of noise, the strategy based on the $\mathbf{b}$ operator fails when $\Delta s$ is greater than $A / 2$ pixels while the strategy based on the optical-flow fails when $\Delta s$ is greater than $A$ pixels. The central remark is that vector $\mathbf{b}$ looks for the nearest edge point. Consequently, given a starting edge point $p$ on frame $f_{n}$, vector $\mathbf{b}$ fails when the point $p$ of frame $f_{n+1}$ is closer to an edge which is not the matching edge $p$ '. On the contrary, the table shows that the optical-flow strategy still works when vector $\mathbf{b}$ fails and continues to work properly until the temporal derivative of eq.(7) does non change its sign, that is, until the displacement is greater than $A$ pixels. Fig. 1 shows three different work conditions: a) both the optical-flow vector $\mathbf{v}$ and vector $\mathbf{b}$ correctly locate the matching edge, $b$ ) the optical-flow vector $\mathbf{v}$ alone correctly locates the matching edge, and c) both vectors $\mathbf{v}$ and $\mathbf{b}$ do not locate the matching edge. The results of table 2 were obtained with a Gaussian noise of variances $v^{2}$ of $225 \mathrm{i.u}$. ${ }^{2}$; a contrast at the step discontinuities of 100 i.u.; $A=10$ pixels; and apertures $\sigma_{l}=2 \sigma_{2}=6$ and $\sigma=6$ pixels. However, in this case also, analogous results were obtained when varying the test parameters.
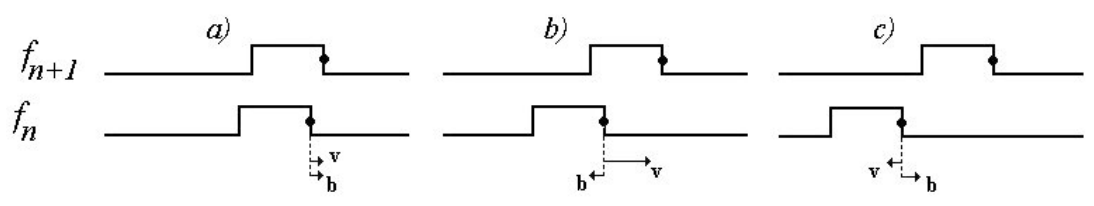

Fig. 1. Three different work conditions are illustrated: a) both the optical-flow vector $\mathbf{v}$ and vector $\mathbf{b}$ correctly locate the matching edge, b) the optical-flow vector $\mathbf{v}$ alone correctly locates the matching edge, and c) both vectors $\mathbf{v}$ and $\mathbf{b}$ do not locate the matching edge

\subsection{Combining the Two Strategies}

The test illustrated in section 4.2 shows that the strategy based on the optical-flow vector should be used to bring a starting edge point of the $\mathrm{n}^{\text {th }}$ frame closer to the respective edge point of the $(n+1)^{\text {th }}$ frame. However, both the tests illustrated in sections 4.1 and 4.2 show that an edge detector should be used to refine the localization process. Consequently, such a localization procedure was used to track the wall of a vessel through sequences of echographic images.

A set of 15 echographic sequences of carotid arteries with a reduced frame rate were used and 50 pairs of consecutive frames $\left(A_{i}\right.$ and $\left.B_{i}\right)$, with a calibration factor of $12 \mathrm{pixel} / \mathrm{mm}$, were chosen. An expert operator traced an approximate contour $a c_{i}$ of the gray level discontinuity generated by the farthest internal wall of the carotid on every $A_{i}$ frame thus obtaining $50 a c_{i}$ contours. Subsequently, vector $\mathbf{b}$ was iteratively applied to every point of $a c_{i}$ in order to locate the true starting contours $s c_{i}$ (each one 20 points long) on frames $A_{i}$. Following that, the two procedures based on the $\mathbf{b}$ vector only and on the optical-flow plus the $\mathbf{b}$ vector, respectively, were applied to every point of the same contours $s c_{i}$. With the first procedure, vector $\mathbf{b}$ was iteratively computed on frames $B_{i}$ using the points of $s c_{i}$ as starting points thus obtaining the final contours $f c_{1 i}$ of frames $B_{i}$. With the second procedure, the optical-flow vector 
was computed on frames $B_{i}$ using the points of $s c_{i}$ as starting points thus locating new starting contours: the middle contours $m c_{i}$. Vector $\mathbf{b}$ was then iteratively computed on frames $B_{i}$ using the points of $m c_{i}$ as starting points to obtain the final contours $f c_{2 i}$ on frames $B_{i}$. Values of $4,0.7$ and 1.4 pixels were used for the apertures $\sigma, \sigma_{1}$ and $\sigma_{2}$, respectively, since the latter proved to be the most appropriate for this task. Fig.2 shows the contours which were located on a pair of $A_{i}$ and $B_{i}$ frames by the two above procedures. When using the strategy based on the $\mathbf{b}$ vector only, a high number of starting points $(80 \%)$ were attracted by adjacent gray level discontinuities. When using the strategy based on the optical-flow plus the b vector the percentage of failures decreased significantly: $10 \%$ only of the starting points were attracted by adjacent gray level discontinuities. The percentage of failures was obtained by counting how many starting points were attracted by adjacent gray level discontinuities which were different from the one generated at the border between the lumen and the farthest internal wall of the carotid. Other configurations (different apertures $\sigma, \sigma_{1}$ and $\sigma_{2}$ ) of the two operators were tested on sequences of vascular images and this additional analysis further supported the previous conclusion: the optical-flow operator alone cannot track contours correctly on long sequences (typically, sequences of 10' must be processed to evaluate the endothelial function) and the $\mathbf{b}$ vector alone may be attracted by adjacent gray level discontinuities (only a few pixels separate the lumen-intima border from the intima-media border). A procedure which uses the two operators sequentially resulted to be a valid choice.

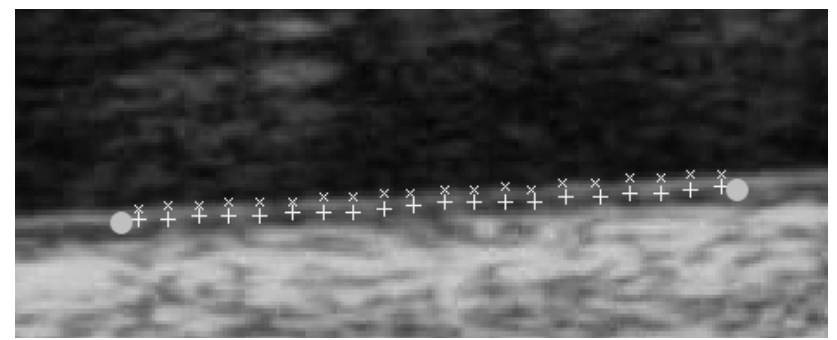

Fig. 2. The frame $f_{n+1}$ of a pair of consecutive frames is shown with superimposed: i) the lumen-intima discontinuity which is given as a starting contour on frame $f_{n}$ (ideal straight line between the two gray spots); ii) the wrong contour which is located by the iterative procedure based on vector $\mathbf{b}$ alone (line of + ); iii) the correct contour which is located by the procedure based on the optical flow plus $\mathbf{b}$ vector (line of $\mathrm{x}$ )

\section{Conclusions}

In this paper only basic operators were used since our objective was to understand their drawbacks: with the exception of Gaussian low-pass filters, neither a true regularization stage nor other possible knowledge based optimizations were purposely exploited. This is also the main reason of our short list of references. Regularization theory and anatomical information can ensure the existence and the uniqueness of the solution as well as its robustness against noise. However, they cannot change the basic principle of a mathematical operator: that is, regularization can improve 
the performances of a procedure, but it cannot eliminate the root of the problem. As an example, a standard block-matching algorithm (block size $31 X 31$ pixels) was also applied to the same test sequences of section 4.1 and a Pratt index ranging from $87 \%$ to $47 \%$ was obtained when varying the number of frames and the displacement $\Delta s$.

Often a pure edge detection strategy does not guarantee the correct tracking of contours and the vascular images are a valuable example of that: here the presence of multiple structures, such as lumen, intima, media, avventitia and tissue, generates multiple gray level discontinuities which are close to each other and the strategy of locating the nearest contour can fail. On the other hand, a strategy which looks for similar gray levels is inappropriate since such a strategy looks for something which is similar and this may be not an edge point. Nevertheless, the drawbacks of the two above strategies can be accepted when the two strategies are used sequentially since they have complementary properties and each of them provides a valid answer to the drawbacks of the other. The strategy which matches regions with similar features can be used to bring a starting contour of a frame close to the respective contour of the subsequent frame and the edge detection strategy can be used to refine the localization process. In this case, an optimization procedure including a global criterion is not necessary since the two strategies can be regularized separately and the computational cost of the contour tracking procedure can be reduced.

\section{References}

1. Schmidt-Trucksass, A., Cheng, D.C., Sandrock, M., Schlte-Monting, J., Rauramaa, R., Huonker, M., Burkhardt, H.: Computerized analysing system using the active contour in ultrasound measurement of carotid artery intima-media thickness. Clinical Physiology 21, $561-569(2001)$

2. Li, M., Kambhamettu, C., Stone, M.: Automatic contour tracking in ultrasound images. Clinical Linguistics and Phonetics 19, 545-554 (2005)

3. Liang, Q., Wendelhag, I., Wikstrand, J., Gustavsson, T.: A Multiscale Dynamic Programming Procedure for Boundary Detection in Ultrasonic Artery Images. IEEE Transaction on Medical Imaging 9(2), 127-142 (2000)

4. Demi, M.: On the Gray-Level Central and Absolute Central Moments and the Mass Center of the Gray-Level Variability in Low Level Image Processing. Computer Vision and Image Understanding 97(2), 180-208 (2005)

5. Beauchemin, S.S., Barron, J.L.: The Computation of Optical Flow. ACM Computing Surveys 27(3), 433-467 (1995)

6. Pratt, W.K.: Digital image processing. Wiley, New York (1991) 